\begin{tabular}{lr}
$\frac{\text { DE }}{6} \begin{array}{l}\text { DE GRUYTER } \\
\text { OPEN }\end{array}$ & $\begin{array}{r}\text { Communications in Applied } \\
\text { and Industrial Mathematics } \\
\text { ISSN 2038-0909 }\end{array}$ \\
Research Article \\
\hline Commun. Appl. Ind. Math. 7 (2), 2016, 8-25 \\
ROI: 10.1515/caim-2016-0003
\end{tabular}

\title{
Electron transport in silicon nanowires having different cross-sections
}

\author{
Orazio Muscato $^{1 *}$, Tina Castiglione ${ }^{1}$ \\ ${ }^{1}$ Dipartimento di Matematica e Informatica \\ Università degli Studi di Catania, Italy \\ *Email address for correspondence: muscato@dmi.unict.it \\ Communicated by Vito Antonio Cimmelli and David Jou \\ Received on January 3, 2015. Accepted on May 7, 2015.
}

\begin{abstract}
Transport phenomena in silicon nanowires with different cross-section are investigated using an Extended Hydrodynamic model, coupled to the Schrödinger-Poisson system. The model has been formulated by closing the moment system derived from the Boltzmann equation on the basis of the maximum entropy principle of Extended Thermodynamics, obtaining explicit closure relations for the high-order fluxes and the production terms. Scattering of electrons with acoustic and non polar optical phonons have been taken into account. The bulk mobility is evaluated for square and equilateral triangle cross-sections of the wire.
\end{abstract}

Keywords: Quantum wires, Semiconductors, Kinetic theory of gases.

AMS subject classification: 82D77, 82D37, 82B40.

\section{INTRODUCTION}

Due to increasing technical difficulties in fabricating planar devices of sub-50 $\mathrm{nm}$ critical dimension, one-dimensional nanowire devices have been suggested as an alternative, to achieve a high stacking density. In fact different electronic structures and transport properties in one dimension can be utilized to fabricate high performance and highly packed integrated circuits (ICs). Sensors, field effect transistors, logic gates and light-emitting devices have been produced using several semiconducting nanowires such as carbon nanotubes, group IV nanowires, and group III-V nanowires [1]. Silicon nanowires (SiNW) are of particular interest since these nanowire devices can be integrated with currently used silicon ICs. In fact, there have been several attempts to fabricate silicon nanowire based devices utilizing conventional silicon processing technology; for example, patterning by lithography, etching and the oxidation of silicon [2]. The electrical transport properties of these wires are particularly important since controllable 


\section{Electron transport in silicon nanowires}

and predictable conductance is vital to many nanoscale electronics applications. By shrinking the dimension of electronic devices, effects of quantum confinement are observed and the wave nature of the electrons must be taken into account. The Non-Equilibrium Green Function formalism is the most advanced transport model for the simulation of SiNW devices, but it necessitates rather intensive computational efforts since it requires detailed information on the propagation of the electron wave packet injected in the device.

Under reasonable hypothesis, transport in low-dimension semiconductors can be tackled coupling quantum and semiclassical tools. In fact, the main quantum transport phenomena in SiNW transistors at room temperature, such as the source-to-drain tunneling, and the conductance fluctuation induced by the quantum interference, become significant only when the longitudinal length (called channel) is smaller than 10nm [3]. Therefore, for longer channels, semiclassical formulations based on the 1-D Multiband Boltzmann Transport Equation (MBTE) can give reliable simulation results when it is solved self-consistently with the 3-D Poisson and 2-D Schrödinger equations in order to obtain the self-consistent potential and subband energies and wavefunctions [1]. Another simplification comes from the use of the Effective Mass Approximation (EMA), which is supposed to be still a good solution in the confining direction in the presence of disorder, which is probably valid for semiconductor nanowires down to $5 \mathrm{~nm}$ in diameter, below which atomistic electronic structure models need to be employed. Solving the MBTE numerically is not an easy task, because it forms an integro-differential system in two dimensions in the phase-space and one in time, with a complicate collisional operator. The full solution of the MBTE can be obtained or by using the Monte Carlo (MC) method [4-9] or by using deterministic numerical solvers [10], [11] at expense of huge computational times. Recently a physics-based hydrodynamic model has been obtained from the MBTE [12-14], taking advantage of the Maximum Entropy Principle.

The aim of this paper is to investigate the transport properties of these nanowires, taking into account the shape of the cross-section. We shall focus ourselves in the square and equilateral triangle cross-sections. In particular quantum wires with equilateral triangle cross-sections have received lots of attention by researchers during the last decade [15], [16], [17], [18], [19] because they represent a step toward the employment of non planar structures for the realization of integrated circuits. 


\section{O. Muscato, T. Castiglione}

\section{Transport equations}

For a quantum wire with linear expansion in z-direction, and confined in the plane $\mathrm{x}-\mathrm{y}$, the normed electron wave function $\psi(x, y, z)$ can be written in the form

$$
\psi(x, y, z)=\chi_{\alpha}(x, y) \frac{e^{i k_{z} z}}{\sqrt{L_{z}}}
$$

where $\chi_{\alpha}(x, y)$ is the wave function of the $\alpha$-th subband and the term $e^{i k_{z} z} / \sqrt{L_{z}}$ describes an independent plane wave in z-direction confined to the normalization length, where $z \in\left[0, L_{z}\right]$ and $k_{z}$ is the wave vector number. In general the electron is subject to external confining potential $U$, such as by a discontinuity in the band gap at an interface between two materials, and also to the effect of the other electrons in the system. The simplest approximation, called Hartree approximation, is to assume that the electrons as whole produce an average electrostatic energy potential $V_{t o t}$, and that a given electron feels the resulting total potential

$$
V_{t o t}=U(x, y)-e \Phi(x, y, z) \quad .
$$

The normed wave function satisfies the Schrödinger equation in the Effective Mass Approximation, i.e.

$$
\left[E_{c}-\frac{\hbar^{2}}{2 m^{*}} \Delta+V_{t o t}(x, y, z)\right] \psi=E \psi
$$

where $E$ is the total energy, $E_{c}$ the conduction band edge energy, and $m^{*}$ denotes the effective mass of the electron in the conduction band. By inserting eq.(1) into eq.(3), in each $z$-th cross section of the device, one obtains the following equation for the envelope function $\chi_{\alpha z}(x, y)$

$\left[-\frac{\hbar^{2}}{2 m^{*}}\left(\frac{\partial^{2}}{\partial x^{2}}+\frac{\partial^{2}}{\partial y^{2}}\right)+U-e \Phi\right] \chi_{\alpha z}=\varepsilon_{\alpha z} \chi_{\alpha z} \quad, \quad E_{\alpha z}=\varepsilon_{\alpha z}+\frac{\hbar^{2} k_{z}^{2}}{2 m^{*}}+E_{c}$

where $\varepsilon_{\alpha z}$ is the kinetic energy associated with the confinement in the $\mathrm{x}-\mathrm{y}$ plane, and we have assumed parabolic band approximation. The term $\Phi$ satisfies the Poisson equation

$$
\nabla \cdot[\epsilon \nabla \Phi(x, y, z)]=e\left(n-N_{D}+N_{A}\right)
$$

where $N_{D}, N_{A}$ are the doping profile (due to donors and acceptors), $\epsilon$ the permittivity, and $n(x, y, z, t)$ is the electron density, which depends on $\chi_{\alpha z}$

$$
n(x, y, z, t)=\sum_{\alpha} \rho^{\alpha}(z, t)\left|\chi_{\alpha z}(x, y, t)\right|^{2}
$$




\section{Electron transport in silicon nanowires}

where $\rho^{\alpha}$ is the subband linear density in the z-direction

$$
\rho^{\alpha}(z, t)=\frac{2}{2 \pi} \int f_{\alpha}\left(z, k_{z}, t\right) d k_{z}
$$

$f_{\alpha}$ being the electron distribution function in the $\alpha$-subband. For an assigned confining potential, one has to solve a coupled problem formed by eqs.(4), (5) and (6) to find $\varepsilon_{\alpha z}, \chi_{\alpha z}$ in each cross-section.

So far, the electrons are free to move in the z-th direction, and can be described using the MBTE [1]

$$
\frac{\partial f_{\alpha}}{\partial t}+v_{z}\left(k_{z}\right) \frac{\partial f_{\alpha}}{\partial z}-\frac{e}{\hbar} \mathcal{E}_{z} \frac{\partial f_{\alpha}}{\partial k_{z}}=\sum_{\alpha^{\prime}} \sum_{\eta} \mathcal{C}_{\eta}\left[f_{\alpha}, f_{\alpha^{\prime}}\right]
$$

where $e$ is the absolute value of the electron charge, $\hbar$ the Plank constant divided by $2 \pi$, and

$$
v_{z}=\frac{1}{\hbar} \frac{\partial E_{\alpha z}}{\partial k_{z}}=\frac{\hbar k_{z}}{m^{*}} \quad, \quad \mathcal{E}_{z}=-\frac{1}{e} \frac{\partial E_{\alpha z}}{\partial z}
$$

are respectively the electron group velocity and the force acting on the electrons. In the low density approximation (not-degenerate case), the collisional operator writes

$$
\mathcal{C}_{\eta}\left[f_{\alpha}, f_{\alpha^{\prime}}\right]=\frac{L_{z}}{2 \pi} \int d k_{z}^{\prime}\left\{w_{\eta}\left(\mathrm{k}^{\prime}, \mathrm{k}\right) f_{\alpha^{\prime}}\left(k_{z}^{\prime}\right)-w_{\eta}\left(\mathrm{k}, \mathrm{k}^{\prime}\right) f_{\alpha}\left(k_{z}\right)\right\}
$$

where $w_{\eta}\left(\mathbf{k}, \mathbf{k}^{\prime}\right)=w_{\eta}\left(\alpha, k_{z}, \alpha^{\prime}, k_{z}^{\prime}\right)$ is the $\eta$-th scattering rate. When $\alpha=\alpha^{\prime}$ we have an intra-subband scattering, otherwise we have an inter-subband scattering.

Scattering mechanisms in SiNW must comprise acoustic phonon scattering (bulk and confined), non-polar optical phonon scattering, surface scattering, scattering with ionized impurities, as well as dielectric screening [5], [6]. However in this preliminary study, for the sake of simplicity, we shall limit ourselves to consider just scattering with optical and acoustic phonons. For the bulk acoustic phonon scattering, in the elastic equipartition approximation, the transition rate is given by [1]

$$
w_{a c}\left(\mathrm{k}, \mathrm{k}^{\prime}\right)=s_{a c} G^{\alpha \alpha^{\prime}} \delta\left(E_{\alpha^{\prime}}-E_{\alpha}\right) \quad, \quad s_{a c}=\frac{2 \pi D_{A}^{2} k_{B} T_{L}}{\rho \hbar v_{s}^{2} L_{z}}
$$

where $D_{A}$ is the acoustic deformation potential $(9 \mathrm{eV}), T_{L}$ the lattice temperature, $\rho$ the mass density $\left(2.33 \mathrm{gr} / \mathrm{cm}^{3}\right), v_{s}$ the sound speed $(6960$ $\mathrm{m} / \mathrm{sec})$, and $G^{\alpha \alpha^{\prime}}$ the confinement factor

$$
G^{\alpha \alpha^{\prime}}=\int\left|\chi_{\alpha^{\prime}}(x, y)\right|^{2}\left|\chi_{\alpha}(x, y)\right|^{2} d x d y
$$




\section{O. Muscato, T. Castiglione}

For the optical phonons we have

$(13)_{o p}\left(\mathrm{k}, \mathrm{k}^{\prime}\right)=s_{o p}\left[g_{0}+\frac{1}{2} \mp \frac{1}{2}\right] G^{\alpha \alpha^{\prime}} \delta\left(E_{\alpha^{\prime}}-E_{\alpha} \mp \hbar \omega_{0}\right) \quad, s_{o p}=\frac{\pi D_{0}^{2}}{\rho \omega_{0} L_{z}}$

where $D_{0}$ is the optical deformation potential $\left(11.410^{8} \mathrm{eV} / \mathrm{cm}\right), \hbar \omega_{0}$ the effective optical phonon energy $(63 \mathrm{meV})$, and $g_{0}$ the Bose-Einstein phonon occupation number.

\section{Extended Hydrodynamic model}

By multiplying the MBTE (8) by the weight functions $\psi_{A}=$ $\left\{1, v_{z}, \varepsilon_{z}, v_{z} \varepsilon_{z}\right\}$, and integrating in the $k_{z}$ space, one obtains the following hydrodynamic-like equations

$$
\begin{aligned}
& \frac{\partial \rho^{\alpha}}{\partial t}+\frac{\partial\left(\rho^{\alpha} V^{\alpha}\right)}{\partial z}=\rho^{\alpha} \sum_{\alpha^{\prime}} C_{\rho}^{\alpha \alpha^{\prime}} \\
& \frac{\partial\left(\rho^{\alpha} V^{\alpha}\right)}{\partial t}+\frac{2}{m^{*}} \frac{\partial\left(\rho^{\alpha} W^{\alpha}\right)}{\partial z}+\frac{e}{m^{*}} \rho^{\alpha} \mathcal{E}_{z}=\rho^{\alpha} \sum_{\alpha^{\prime}} C_{V}^{\alpha \alpha^{\prime}} \\
& \frac{\partial\left(\rho^{\alpha} W^{\alpha}\right)}{\partial t}+\frac{\partial\left(\rho^{\alpha} S^{\alpha}\right)}{\partial z}+\rho^{\alpha} e \mathcal{E}_{z} V^{\alpha}=\rho^{\alpha} \sum_{\alpha^{\prime}} C_{W}^{\alpha \alpha^{\prime}} \\
& \frac{\partial\left(\rho^{\alpha} S^{\alpha}\right)}{\partial t}+\frac{\partial\left(\rho^{\alpha} F^{\alpha}\right)}{\partial z}+3 \frac{e}{m^{*}} \rho^{\alpha} \mathcal{E}_{z} W^{\alpha}=\rho^{\alpha} \sum_{\alpha^{\prime}} C_{S}^{\alpha \alpha^{\prime}}
\end{aligned}
$$

in the unknowns (called moments)

(18) $\quad V^{\alpha}=\frac{2}{(2 \pi)} \frac{1}{\rho^{\alpha}} \int_{\mathbb{R}} f_{\alpha}\left(z, k_{z}, t\right) v_{z} d k_{z} \quad$ (subband velocity),

(19) $\quad W^{\alpha}=\frac{2}{(2 \pi)} \frac{1}{\rho^{\alpha}} \int_{\mathbb{R}} f_{\alpha}\left(z, k_{z}, t\right) \varepsilon_{z} d k_{z} \quad$ (subband energy),

(20) $\quad S^{\alpha}=\frac{2}{(2 \pi)} \frac{1}{\rho^{\alpha}} \int_{\mathbb{R}} f_{\alpha}\left(z, k_{z}, t\right) \varepsilon_{z} v_{z} d k_{z} \quad$ (subband energy- flux) 


\section{Electron transport in silicon nanowires}

and the higher-order flux $F^{\alpha}$, and the production terms

$$
\begin{aligned}
& F^{\alpha}=\frac{2}{(2 \pi)} \frac{1}{\rho^{\alpha}} \int f_{\alpha} v_{z}^{2} \varepsilon_{z} d k_{z} \\
& C_{\rho}^{\alpha \alpha^{\prime}}=\frac{2}{(2 \pi)} \frac{1}{\rho^{\alpha}} \sum_{\eta} \int \mathcal{C}_{\eta}\left[f_{\alpha}, f_{\alpha^{\prime}}\right] d k_{z} \\
& C_{V}^{\alpha \alpha^{\prime}}=\frac{2}{(2 \pi)} \frac{1}{\rho^{\alpha}} \sum_{\eta} \int \mathcal{C}_{\eta}\left[f_{\alpha}, f_{\alpha^{\prime}}\right] v_{z} d k_{z} \\
& C_{W}^{\alpha \alpha^{\prime}}=\frac{2}{(2 \pi)} \frac{1}{\rho^{\alpha}} \sum_{\eta} \int \mathcal{C}_{\eta}\left[f_{\alpha}, f_{\alpha^{\prime}}\right] \varepsilon_{z} d k_{z} \\
& C_{S}^{\alpha \alpha^{\prime}}=\frac{2}{(2 \pi)} \frac{1}{\rho^{\alpha}} \sum_{\eta} \int \mathcal{C}_{\eta}\left[f_{\alpha}, f_{\alpha^{\prime}}\right] \varepsilon_{z} v_{z} d k_{z} .
\end{aligned}
$$

This system of PDEs is of hyperbolic type and it is not closed, i.e. there are more unknowns than equations. The Maximum Entropy Principle leads to a systematic way for obtaining constitutive relations on the basis of the information theory [20], as already proved successfully in the bulk case [2125], and for quantum well structures [26], [27]. Actually, in a semiconductor electrons interact with phonons describing the thermal vibrations of the ions placed at the points of the crystal lattice. However, since we are considering the phonon gas as a thermal bath, one has to extremize only the electron component of the entropy. We define the entropy of the electronic system as

$$
\begin{aligned}
& S_{e}=\sum_{\alpha}\left|\chi_{\alpha}(x, y, t)\right|^{2} S_{e}^{\alpha} \\
& S_{e}^{\alpha}=-\frac{2}{(2 \pi)} k_{B} \int_{\mathbb{R}}\left(f_{\alpha} \log f_{\alpha}-f_{\alpha}\right) d k_{z},
\end{aligned}
$$

and, according to MEP, we estimate the $f_{\alpha}$ 's as the distributions that maximize $S_{e}$ under the constraints that the basic moments, which we have previously considered, are assigned. In a neighborhood of local thermal equilibrium, this distribution function writes [12]

$$
\hat{f}_{\alpha}=\exp \left(-\frac{\lambda^{\alpha}}{k_{B}}-\lambda_{W}^{\alpha} \varepsilon_{z}\right)\left\{1-\tau\left(\hat{\lambda}_{V}^{\alpha} v_{z}+\hat{\lambda}_{S}^{\alpha} v_{z} \varepsilon_{z}\right)\right\}
$$

where the quantities $\left(\lambda^{\alpha}, \lambda_{W}^{\alpha}, \hat{\lambda}_{V}^{\alpha}, \hat{\lambda}_{S}^{\alpha}\right)$ are known functions of the moments $\left\{\rho^{\alpha}, V^{\alpha}, W^{\alpha}, S^{\alpha}\right\}$. By using the distribution function (28) it is possible to evaluate the unknown functions appearing in the balance equations by integration. In this way the higher-order flux term writes 


$$
F^{\alpha}=\frac{6\left(W^{\alpha}\right)^{2}}{m^{*}}
$$

as well as the production terms $C_{\rho}^{\alpha \alpha^{\prime}}, C_{V}^{\alpha \alpha^{\prime}}, C_{W}^{\alpha \alpha^{\prime}}, C_{S}^{\alpha \alpha^{\prime}}$ have been determined in [12]. We want underline that this Extended Hydrodynamic model has been closed by using first principles, and it is free of any fitting parameters.

\section{Electron Mobility}

The mobility is one of the most important parameters that determine the performance of a field-effect transistor. At low electric field, the carrier drift velocity is proportional to the electric field strength, and the proportionality constant is defined as the mobility. Hence a higher mobility material is likely to have higher frequency response, because carriers take less time to travel through the device. When the fields are sufficiently large, nonlinearities in the mobility and saturation in the drift velocity are observed. In fact, the scattering of the carriers with the lattice, the impurities, and the surface is more active for higher fields, and the charges lose the energy gained by the electric field.

We shall assume that the cross-section $A$ of the wire is surrounded by an oxide which gives rise to an infinitely deep potential barrier,

$$
U(x, y)= \begin{cases}0 & (x, y) \in A \\ \infty & \text { otherwise }\end{cases}
$$

In such a case analytical relations for the confinement energies $\varepsilon_{\alpha z}$ and envelope functions $\chi_{\alpha z}$ can be obtained from eq.(4) (with $\Phi=0$ ), according to the shape of the cross-section:

- Rectangular cross-section.

In this case, after simple calculations, one obtains [1]

$$
\begin{aligned}
& \text { (32) } \chi_{m, n}(x, y)=\sqrt{\frac{2}{L_{x}}} \sin \left(\frac{n \pi}{L_{x}} x\right) \sqrt{\frac{2}{L_{y}}} \sin \left(\frac{m \pi}{L_{y}} y\right) \\
& \left(33 g_{r}^{\alpha \alpha^{\prime}}=\frac{1}{L_{x} L_{y}}\left[1+\frac{1}{2} \frac{\sin \left(\left(n-n^{\prime}\right) \pi\right)}{\left(n-n^{\prime}\right) \pi}\right]\left[1+\frac{1}{2} \frac{\sin \left(\left(m-m^{\prime}\right) \pi\right)}{\left(m-m^{\prime}\right) \pi}\right]\right.
\end{aligned}
$$




\section{Electron transport in silicon nanowires}

where $L_{x}, L_{y}$ are the rectangle dimensions, and $\alpha=(m, n), \alpha^{\prime}=$ $\left(m^{\prime}, n^{\prime}\right)$.

- Equilateral triangle cross-section.

Let be $L$ the length of the triangle side. Using group theory arguments one obtains [28]

$$
\begin{gathered}
\varepsilon_{p, q}(34) \frac{8 \hbar^{2} \pi^{2}}{3 m^{*} L^{2}}\left(p^{2}+p q+q^{2}\right), q=0,1,2, . . \quad, p=q+1, q+2, q+3, . . \\
(35) \chi_{p, 0}(x, y)=\frac{\sqrt{8}}{3^{\frac{3}{4}} L} \times \\
\quad\left[\sin \left(\frac{4 p \pi y}{\sqrt{3} L}\right)-2 \sin \left(\frac{2 p \pi y}{\sqrt{3} L}\right) \cos \left(\frac{2 p \pi x}{L}\right)\right], p=1,2,3, \ldots \\
(36) \quad \chi_{p, q}(x, y)=\frac{4}{3^{\frac{3}{4}} L}\left\{\cos \left[\frac{2 \pi x q}{L}\right] \sin \left[\frac{2 \pi y(2 p+q)}{\sqrt{3} L}\right]-\right. \\
\left.\cos \left[\frac{2 \pi x p}{L}\right] \sin \left[\frac{2 \pi y(2 q+p)}{\sqrt{3} L}\right]-\cos \left[\frac{2 \pi x(p+q)}{L}\right] \sin \left[\frac{2 \pi y(p-q)}{\sqrt{3} L}\right]\right\} \\
q=1,2,3, \ldots, p=q+1, q+2, q+3, \ldots
\end{gathered}
$$

Let us define $\alpha=(p, q), \alpha^{\prime}=\left(p^{\prime}, q^{\prime}\right)$ then, after long but straightforward calculations, one obtains:

$$
\begin{aligned}
& \text { - if } p \neq p^{\prime} \text { and } p^{\prime} \neq 2 p \text { then } G^{\alpha \alpha^{\prime}}=\frac{4}{3} \frac{\sqrt{3}}{L^{2}} ; \\
& \text { - if } p=p^{\prime} \text { then } G^{\alpha \alpha^{\prime}}=\frac{10}{3} \frac{\sqrt{3}}{L^{2}} ; \\
& \text { - If } p^{\prime}=2 p \text { the } G^{\alpha \alpha^{\prime}}=\frac{16}{9} \frac{\sqrt{3}}{L^{2}} .
\end{aligned}
$$

In the following we shall compare two SiNW having square and equilateral triangle cross-sections, in such a way both have the same area $(A=100$ $\mathrm{nm}^{2}$ ). In particular we have chosen $L_{x}=L_{y}=10 \mathrm{~nm}$ and $L=20 / \sqrt[4]{3} \simeq$ $15.19 \mathrm{~nm}$. In the figure 1 we plot the lowest four confinement energies for the square and equilateral triangle cross-sections. We observe that the energy levels are higher in the triangular case.

We want to obtain the drift velocity and the mobility in the so called bulk case. We shall assume that the SiNW is homogeneous and sufficiently long in such a way the neutrality charge condition hold, i.e. $n-N_{D}+N_{A}=0$. Then, by assuming Neumann boundary conditions at the oxide interface, and Dirichelet boundary conditions at the cathode and anode

$$
\left.\frac{\partial \Phi}{\partial \mathbf{n}}\right|_{\partial A}=0 \quad, \quad \Phi(x, y, 0)=0 \quad, \quad \Phi\left(x, y, L_{z}\right)=e V_{b}
$$




\section{O. Muscato, T. Castiglione}

where $\mathbf{n}$ is the unit vector normal to $\partial A$, the Poisson equation (5) gives

$$
\Phi(x, y, z)=\frac{e V_{b}}{L_{z}} z
$$

The EME equation (4) with (30) and (38), gives

$$
E_{\alpha z}=\varepsilon_{\alpha z}+\frac{e V_{b}}{L_{z}} z+\frac{\hbar^{2} k_{z}^{2}}{2 m^{*}}+E_{c}
$$

where the confinement energies $\varepsilon_{\alpha z}$ and the envelope functions $\chi_{\alpha z}$ are given by the previous formulas (31)-(36), and the force acting of the electrons $(9)_{2}$ reduces to the usual electric field

$$
\mathcal{E}_{z}=-\frac{1}{e} \frac{\partial E_{\alpha z}}{\partial z}=-\frac{V_{b}}{L_{z}}
$$

We have performed a numerical integration of our hydrodynamic model in the stationary homogeneous case with a constant electric field $\mathcal{E}_{z}$ along the $z$ direction. In this case the unknowns $\left(\rho^{\alpha}, V^{\alpha}, W^{\alpha}, S^{\alpha}\right)$ depend on the time only. The initial data are the equilibrium values obtained with a global Maxwellian i.e.

$$
f_{M}^{\alpha}\left(k_{z}\right)=\exp \left(-\frac{\frac{\hbar^{2} k_{z}^{2}}{2 m^{*}}+\varepsilon_{\alpha z}+E_{c}-\nu^{\alpha}}{k_{B} T_{0}^{\alpha}}\right)
$$

where $\nu^{\alpha}$ is the equilibrium chemical potential, and $T_{0}(300 \mathrm{~K})$ the lattice temperature. By inserting the eq.(41) into (7), (18)-(20) after simple calculation we get

$$
\begin{gathered}
V^{\alpha}(0)=0 \quad, W^{\alpha}(0)=\frac{1}{2} k_{B} T_{0} \quad, S^{\alpha}(0)=0 \\
\rho^{\alpha}(0)=A N_{D} \frac{\exp \left(-\frac{\varepsilon_{\alpha}}{k_{B} T_{0}}\right)}{\sum_{\alpha} \exp \left(-\frac{\varepsilon_{\alpha}}{k_{B} T_{0}}\right)} .
\end{gathered}
$$

The numerical experiments indicate that it is sufficient to take into account only the first four subbands $(\alpha=1,2,3,4)$, since the other ones are very scarcely populated. The average drift velocity is defined as

$$
\langle V(t)\rangle=\frac{\sum_{\alpha} \rho^{\alpha} V^{\alpha}}{\sum_{\alpha} \rho^{\alpha}} .
$$

In the figure 2 we plot the subband velocities $V^{\alpha}(t)(\alpha=1, \ldots, 4)$ as well as the average drift velocity versus the simulation time, for an electric field of 


\section{Electron transport in silicon nanowires}

$8000 \mathrm{~V} / \mathrm{cm}$, and the equilateral triangle cross-section. During the transient period the average velocity overshoots its asymptotic value, that is the value attained in the stationary case. For the same case, in the figure 3 , we plot the subband linear densities: the linear density in the forth subband is about $5 \%$ of the total linear density as a confirmation that the inclusion of further subbands has a negligible effect.

Then we have changed the electric field and plotted, in the figure 4, the corresponding asymptotic value of the velocity $\langle V(\infty)\rangle$ : the typical saturation effect is shown. The bulk mobility is defined as

$$
\mu\left(\mathcal{E}_{z}\right)=\frac{\sum_{\alpha} \rho^{\alpha} \mu^{\alpha}}{\sum_{\alpha} \rho^{\alpha}} \quad, \quad \mu^{\alpha}=\frac{V^{\alpha}(\infty)}{\mathcal{E}_{z}}
$$

where $\mu^{\alpha}$ is the subband mobility. In the figures 5,6 we plot the bulk mobility as function of the electric field for the triangular and square crosssections. In the same figures, we have compared the mobility obtained by means of our hydrodynamic model, with the mobility given by the CaugheyThomas formula [29]

$$
\mu_{C}=\mu_{0}\left[1+\left(\frac{\mu_{0} \mathcal{E}_{z}}{v_{s}}\right)^{2}\right]^{-\frac{1}{2}}
$$

where $\mu_{0}=\mu\left(\mathcal{E}_{z}=10 \mathrm{~V} / \mathrm{cm}\right)$ and $v_{s}$ is the average drift velocity for high fields. From the above figures we notice that, for low fields $(\leq 1000 \mathrm{~V} / \mathrm{cm})$, the mobility is constant whereas, for high fields, the mobility decreases because the scattering processes become more active. Similar results have been obtained, in the square case, using the more expensive MC simulations in [5]. We notice also that the value obtained for the low field mobility in the triangular cross-section case $\left(\mu_{0}=1600 \mathrm{~cm}^{2} \mathrm{~V} / \mathrm{sec}\right)$ is bigger than that obtained in the square case $\left(\mu_{0}=406 \mathrm{~cm}^{2} \mathrm{~V} / \mathrm{sec}\right)$, and this value is closer to the measured value for bulk silicon without any confinement effect $\left(1450 \mathrm{~cm}^{2} \mathrm{~V} / \mathrm{sec}\right.$ [30]). In order to explain this behaviour, we notice that the confinement factor $G^{\alpha \alpha^{\prime}}$ is smaller in the triangular case with respect to the square case. Then the scattering rates (11), (13) are reduced and that results in an enhancement of the mobility. The values of the mobilities found can be altered consistently if we take into account other relevant physical effects such as the modification of the phonon spectrum due to spatial confinement [5], and the surface roughness and impurity scattering.

\section{Conclusions}

An extended hydrodynamic model for SiNW has been formulated with the use of the maximum entropy principle, where the transport coefficients 


\section{O. Muscato, T. Castiglione}

are completely determined without any fitting procedure. The bulk electron mobility (low and high-field) has been evaluated for SiNW with square and equilateral triangle cross-sections having the same area. In particular we have found higher mobility values in the triangular case. This behaviour can be justified by a decrease in the scattering rate, due to the confinement factor. In order to confirm the goodness of the triangular cross-section, our model must be improved by including other relevant scattering mechanisms such as scattering with impurities and surface roughness, as well as to take into account the modification of the acoustic phonon spectrum in such nanostructures. These topics as well as the study of thermoelectric effects according to the guideline in [31-38] will be the subjects of future researches.

\section{Acknowledgment}

We acknowledge the support of the Università degli Studi di Catania, FIR 2014 "Charge Transport in Graphene and Low dimensional Structures: modeling and simulation". 


\section{Electron transport in silicon nanowires}

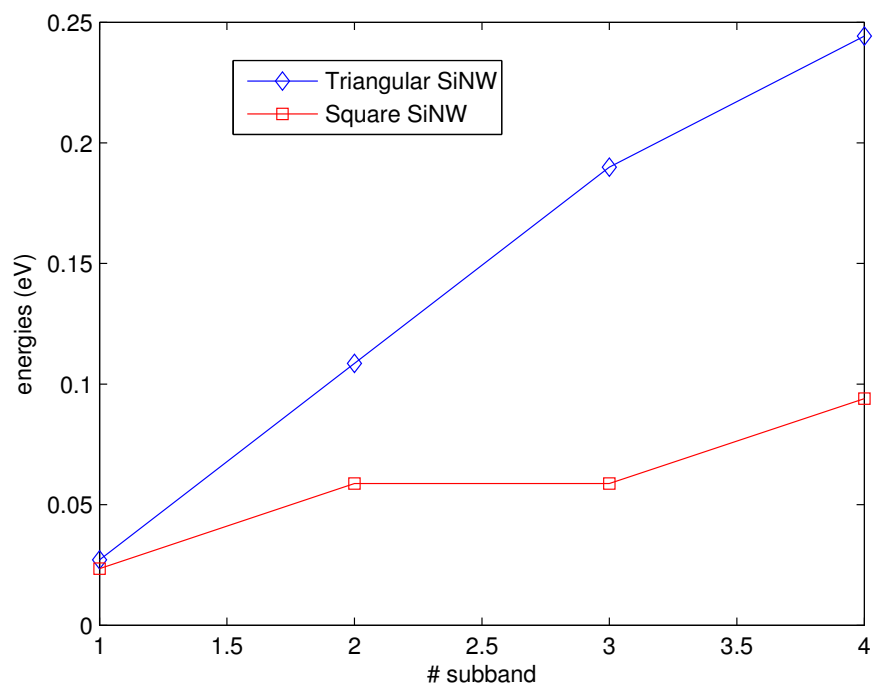

Figure 1. The lowest four confinement energies for the square and equilateral triangle cross-sections.

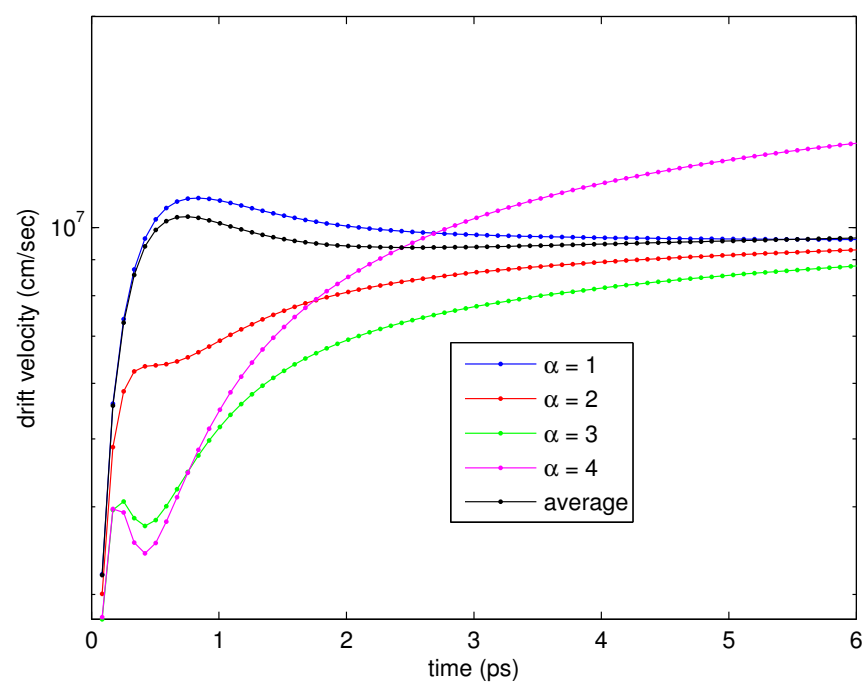

Figure 2. The subband drift velocities $V^{\alpha}(t)$ and the average drift velocity (44) versus the simulation time, for an electric field of $8000 \mathrm{~V} / \mathrm{cm}$, and the equilateral triangle crosssection. 
O. Muscato, T. Castiglione

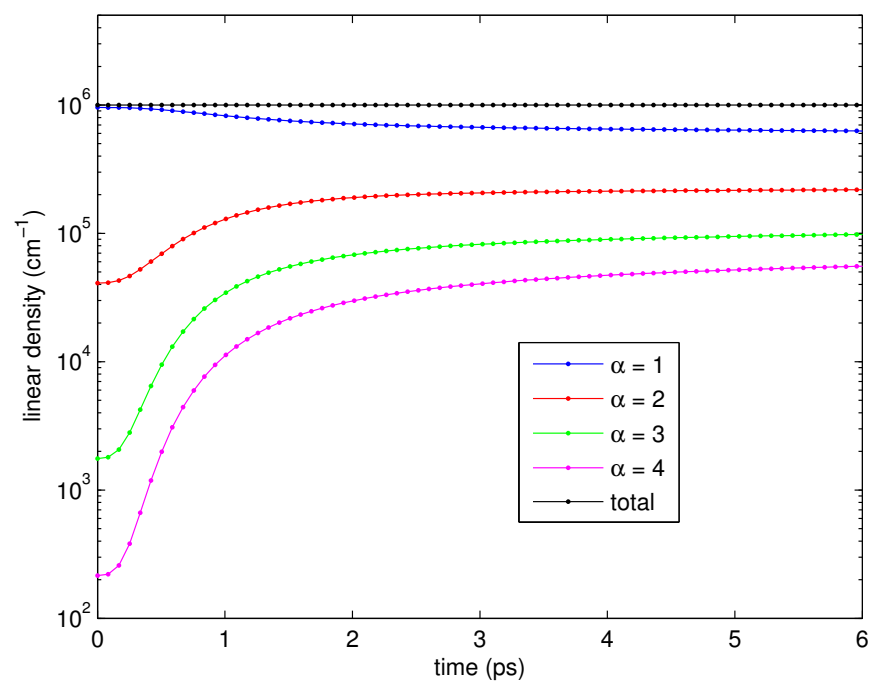

Figure 3. The subband linear densities $\rho^{\alpha}$ and the total linear density $\left(\sum_{\alpha} \rho^{\alpha}\right)$ versus the simulation time, for an electric field of $8000 \mathrm{~V} / \mathrm{cm}$, and the the equilateral triangle cross-section. 


\section{Electron transport in silicon nanowires}

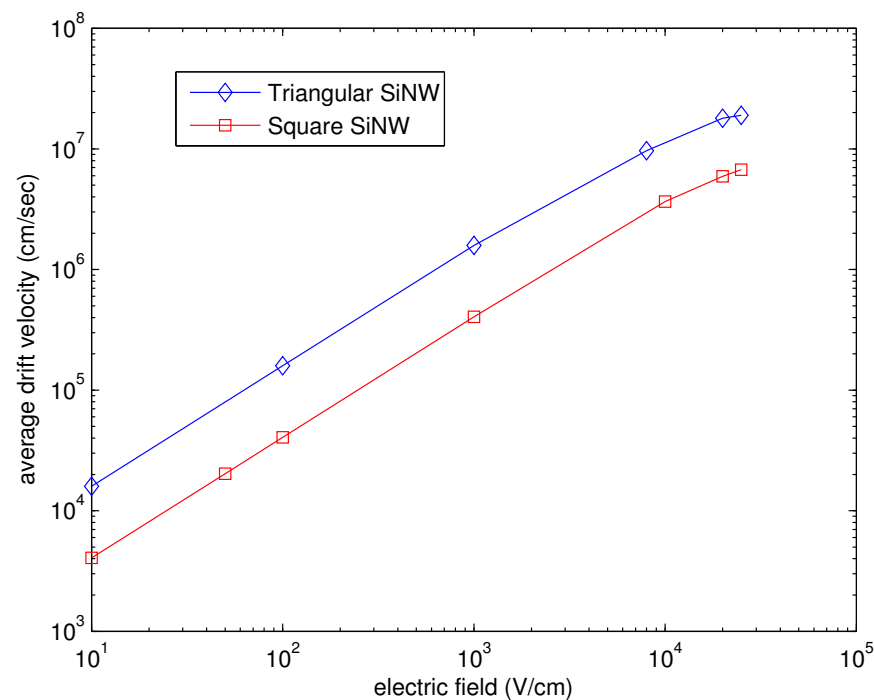

Figure 4. The asymptotic value of the average velocity $\langle V(\infty)\rangle(44)$ versus the electric field for the square and the equilateral triangle cross-sections.

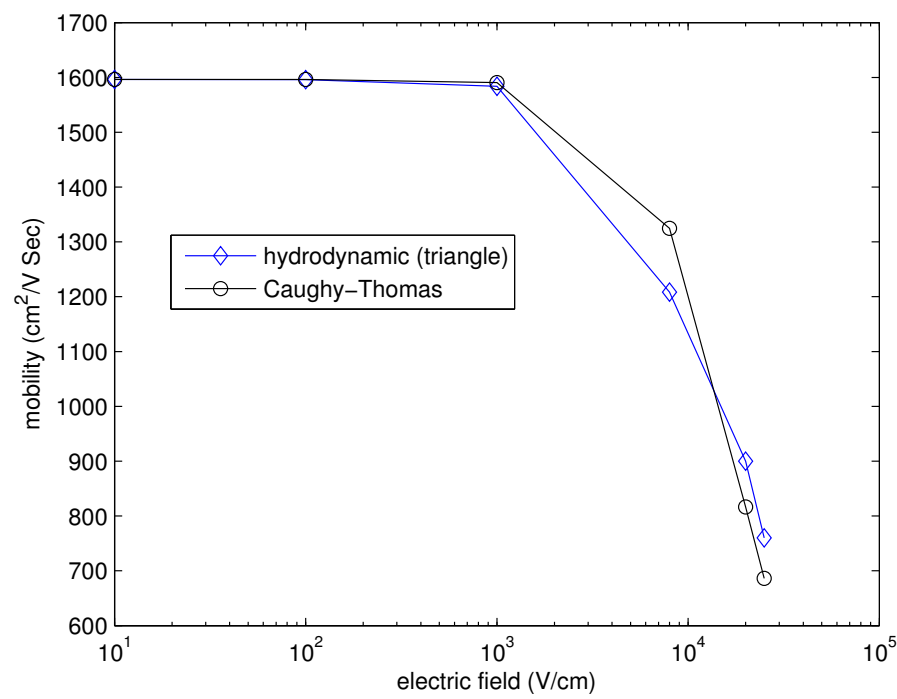

Figure 5. The bulk mobility 45) versus the electric field for the equilateral triangle cross-section, and the mobility evaluated using the Caughey-Thomas formula (46). 


\section{O. Muscato, T. Castiglione}

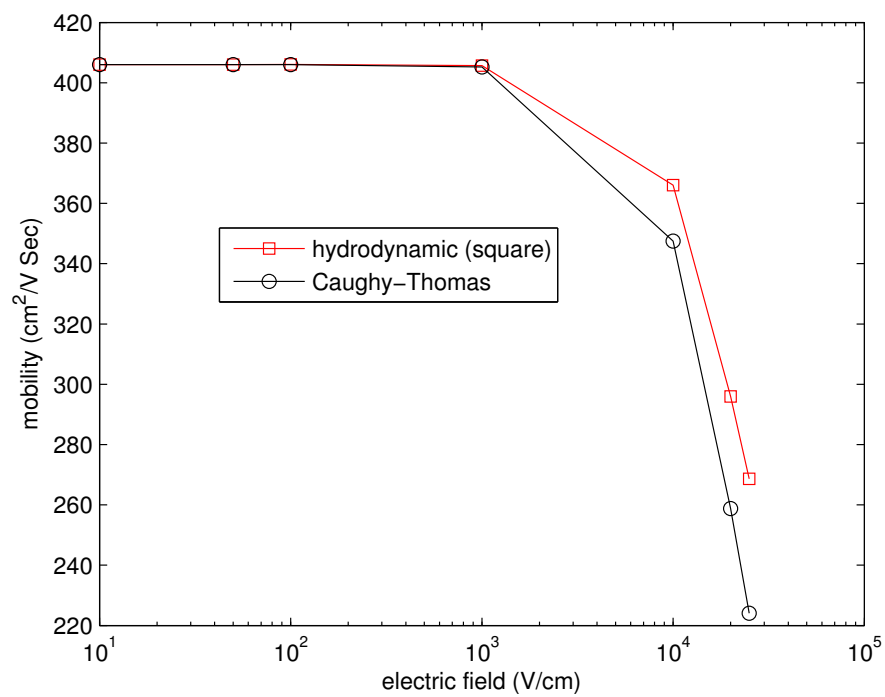

Figure 6. The average mobility $(45)_{1}$ (circles) versus the electric field for the square cross-section, and the mobility evaluated using the Caughey-Thomas formula (46).

\section{REFERENCES}

1. D. Ferry, S. Goodnick, and J. Bird, Transport in nanostructures. Cambridge University Press, 2009.

2. R. Juhasz, N. Elfstro, and J. Linnros, Controlled fabrication of silicon nanowires by electron beam lithography and electrochemical size reduction, Nano Letters, vol. 5, no. 2, pp. 275-280, 2005.

3. M. Lundstrom and J. Wang, Does source-to-drain tunneling limit the ultimate scaling of mosfets?, IEDM Tech. Dig., pp. 707-710, 2002.

4. E. Ramayya, D. Vasileska, S. Goodnick, and I. Knezevic, Electron mobility in silicon nanowires, IEEE Trans. Nanotech., vol. 6, no. 1, pp. 113$117,2007$.

5. E. Ramayya, D. Vasileska, S. Goodnick, and I. Knezevic, Electron transport in silicon nanowires: The role of acoustic phonon confinement and surface roughness scattering, J. Appl. Phys., vol. 104, p. 063711, 2008.

6. E. Ramayya and I. Knezevic, Self-consistent Poisson-Schrödinger-Monte Carlo solver: electron mobility in silicon nanowires, J. Comput. Electr., vol. 9, pp. 206-210, 2010.

7. O. Muscato, W. Wagner, and V. Di Stefano, Numerical study of the systematic error in Monte Carlo schemes for semiconductors, ESAIM: M2AN, vol. 44, no. 5, pp. 1049-1068, 2010.

8. O. Muscato, W. Wagner, and V. Di Stefano, Properties of the steady 


\section{Electron transport in silicon nanowires}

state distribution of electrons in semiconductors, Kinetic and Related Models, vol. 4, no. 3, pp. 809-829, 2011.

9. O. Muscato, V. Di Stefano, and W. Wagner, A variance-reduced electrothermal Monte Carlo method for semiconductor device simulation, Comput. Math. with Appl., vol. 65, no. 3, pp. 520-527, 2013.

10. M. Lenzi, P. Palestri, E. Gnani, A. Gnudi, D. Esseni, L. Selmi, and G. Baccarani, Investigation of the transport properties of silicon nanowires using deterministic and Monte Carlo approaches to the solution of the boltzmann transport equation, IEEE Trans. Electr. Dev., vol. 55 , no. 8, pp. 2086-2096, 2008.

11. G. Ossig and F. Schuerrer, Simulation of non-equilibrium electron transport in silicon quantum wires, J. Comput. Electron., vol. 7, pp. 367-370, 2008.

12. O. Muscato and V. Di Stefano, Hydrodynamic modeling of silicon quantum wires, J. Comput. Electron., vol. 11, no. 1, pp. 45-55, 2012.

13. V. Di Stefano and O. Muscato, Seebeck effect in silicon semiconductors, Acta Appl. Math., vol. 122, no. 1, pp. 225-238, 2012.

14. O. Muscato and V. Di Stefano, Hydrodynamic simulation of a $\mathrm{n}+-\mathrm{n}-$ n+ silicon nanowire, Contin. Mech. Thermodyn., vol. 26, pp. 197-205, 2014.

15. T. Kuykendall, P. Pauzauskie, S. Lee, Y. Zhang, J. Goldberger, and P. Yang, Metalorganic chemical vapor deposition route to gan nanowires with triangular cross sections, Nano Letters, vol. 3, no. 8, pp. 1063-1066, 2003.

16. G. Pennelli and M. Piotto, Fabrication and characterization of silicon nanowires with triangular cross section, J. Appl. Phys., vol. 100, p. 054507, 2006.

17. G. Pennelli, Top down fabrication of long silicon nanowire devices by means of lateral oxidation, Microelec. Engineer., vol. 86, pp. 2139-2143, 2009.

18. G. Liang, W. Huang, C. S. Koong, J.-S. Wang, and J. Lan, Geometry effects on thermoelectric properties of silicon nanowires based on electronic band structures, J. Appl. Phys., vol. 107, p. 014317, 2010.

19. R. Khordad and H. Bahramiyan, Electron-phonon interaction effect on the energy levels and diamagnetic susceptibility of quantum wires: Parallelogram and triangle cross section, J. Appl. Phys., vol. 115, p. 124314, 2014.

20. D. Jou, J. Casas-Vázquez, and G. Lebon, Extended irreversible thermodynamics. Springer-Verlag, 2001.

21. O. Muscato, R. Pidatella, and M. Fischetti, Monte Carlo and hydrodynamic simulation of a one dimensional $n^{+}-n-n^{+}$silicon diode, VLSI 


\section{O. Muscato, T. Castiglione}

Design, vol. 6, no. 1-4, pp. 247-250, 1998.

22. O. Muscato and V. Di Stefano, Modeling heat generation in a submicrometric $n^{+}-n-n^{+}$silicon diode, J. Appl. Phys., vol. 104, no. 12, p. $124501,2008$.

23. O. Muscato and V. Di Stefano, Hydrodynamic modeling of the electrothermal transport in silicon semiconductors, J. Phys. A: Math. Theor., vol. 44, no. 10, p. 105501, 2011.

24. O. Muscato and V. Di Stefano, An energy transport model describing heat generation and conduction in silicon semiconductors, J. Stat. Phys., vol. 144, no. 1, pp. 171-197, 2011.

25. O. Muscato and V. Di Stefano, Heat generation and transport in nanoscale semiconductor devices via Monte Carlo and hydrodynamic simulations, COMPEL, vol. 30, no. 2, pp. 519-537, 2011.

26. G. Mascali and V. Romano, A non parabolic hydrodynamical subband model for semiconductors based on the maximum entropy principle, Math. Comp. Model., vol. 55, no. 3-4, pp. 1003-1020, 2012.

27. V. Camiola, G. Mascali, and V. Romano, Numerical simulation of a double-gate mosfet with a subband model for semiconductors based on the maximum entropy principle, Contin. Mech.Thermodyn., vol. 24, no. 4-6, pp. 417-436, 2012.

28. W.-K. Li and S. Blinder, Solution of the Schrödinger equation for a particle in an equilateral triangle, J. Math. Phys., vol. 26, no. 11, pp. 2784$2786,1985$.

29. S. Selberherr, Analysis and Simulation of Semiconductor Devices. Springer, 1984.

30. C. Jacoboni, C. Canali, G. Ottaviani, and A. Quaranta, A review of some charge transport properties for silicon, Solid State Electron, vol. 20, no. 2, pp. 77-89, 1977.

31. O. Muscato and V. Di Stefano, Local equilibrium and off-equilibrium thermoelectric effects in silicon semiconductors, J. Appl. Phys., vol. 110, no. 9, p. 093706, 2011.

32. O. Muscato and V. Di Stefano, Electro-thermal behaviour of a submicron silicon diode, Semicond. Sci. Tech., vol. 28, no. 2, p. 025021, 2013.

33. E. Ramayya, L. Maurer, A. Davoody, and I. Knezevic, Thermoelectric properties of ultrathin silicon nanowires, Phys. Rev. B, vol. 86, no. 11, p. 115328, 2012.

34. Z. Aksamija and I. Knezevic, Thermoelectric properties of properties of silicon nanostructures, J. Comput. Electron., vol. 9, pp. 173-179, 2010.

35. D. Jou, V. Cimmelli, and A. Sellito, Nonlocal heat transport with phonons and electrons: Application to metallic nanowires, Int. J. Heat 


\section{Electron transport in silicon nanowires}

Mass transf., vol. 55, no. 9-10, pp. 2338-2344, 2012.

36. A. Sellito, V. Cimmelli, and D. Jou, Thermoelectric effects and size dependency of the figure-of-merit in cylindrical nanowires, Int. J. Heat Mass transf., vol. 57, no. 1, pp. 109-116, 2013.

37. V. Cimmelli, A. Sellito, and D. Jou, A nonlinear thermodynamic model for a breakdown of the onsager symmetry and the efficiency of thermoelectric conversion in nanowires, Proc. Royal soc.A: Math., Phys. Eng. Sci., vol. 470, no. 2170, p. 20140265, 2014.

38. A. Sellito and V. Cimmelli, Flux limiters in radial heat transport in silicon nanolayers, J. Heat Transfer, vol. 136, no. 7, p. 071301, 2014. 